\title{
Identification of Potential Virulence Factors in the Model Strain Acinetobacter baumannii A118
}

\author{
Maria S. Ramirez ${ }^{1}$, William F. Penwell ${ }^{2}$, German M. Traglia ${ }^{3}$, Daniel L. Zimbler², \\ Jennifer A. Gaddy2,4, Nikolas Nikolaidis ${ }^{1}$, Brock A. Arivett ${ }^{2}$, Mark D. Adams ${ }^{5}$, \\ Robert A. Bonomo ${ }^{6}$, Luis A. Actis ${ }^{2}$ and Marcelo E. Tolmasky ${ }^{1 *}$
}

OPEN ACCESS

Edited by:

Miklos Fuzi,

Semmelweis University, Hungary

Reviewed by:

Andres Felipe Opazo-Capurro, Universidad de Concepción, Chile

Paolo Visca,

Roma Tre University, Italy

Lenie Dijkshoorn,

Leiden University Medical Center,

Netherlands

*Correspondence:

Marcelo E. Tolmasky

mtolmasky@fullerton.edu

Specialty section:

This article was submitted to

Infectious Diseases,

a section of the journal

Frontiers in Microbiology

Received: 01 March 2019

Accepted: 26 June 2019

Published: 23 July 2019

Citation:

Ramirez MS, Penwell WF,

Traglia GM, Zimbler DL, Gaddy JA, Nikolaidis N, Arivett BA, Adams MD,

Bonomo RA, Actis LA and Tolmasky ME (2019) Identification

of Potential Virulence Factors in the Model Strain Acinetobacter

baumannii A118.

Front. Microbiol. 10:1599. doi: 10.3389/fmicb.2019.01599

\begin{abstract}
${ }^{1}$ Department of Biological Science, Center for Applied Biotechnology Studies, California State University, Fullerton, Fullerton, CA, United States, ${ }^{2}$ Department of Microbiology, Miami University, Oxford, OH, United States, ${ }^{3}$ Laboratorio de Bacteriología Clínica, Departamento de Bioquímica Clínica, Facultad de Farmacia y Bioquímica, Hospital de Clínicas "José de San Martín", Buenos Aires, Argentina, ${ }^{4}$ Department of Medicine, Vanderbilt University Medical Center, Nashville, TN, United States,

${ }^{5}$ Department of Genetics, School of Medicine, Case Western Reserve University, Cleveland, OH, United States,

${ }^{6}$ Departments of Pharmacology and Molecular Biology and Microbiology, Louis Stokes Cleveland Veterans Affairs Medical Center, School of Medicine, Case Western Reserve University, Cleveland, OH, United States
\end{abstract}

Acinetobacter baumannii A118, a strain isolated from the blood of an infected patient, is naturally competent and unlike most clinical strains, is susceptible to a variety of different antibiotics including those usually used for selection in genetic manipulations. These characteristics make strain A118 a convenient model for genetic studies of A. baumannii. To identify potential virulence factors, its complete genome was analyzed and compared to other $A$. baumannii genomes. A. baumannii A118 includes gene clusters coding for the acinetobactin and baumannoferrin iron acquisition systems. Ironregulated expression of the BauA outer membrane receptor for ferric-acinetobactin complexes was confirmed as well as the utilization of acinetobactin. A. baumannii A118 also possesses the feoABC genes, which code for the main bacterial ferrous uptake system. The functionality of baumannoferrin was suggested by the ability of A. baumannii A118 culture supernatants to cross feed an indicator BauA-deficient strain plated on iron-limiting media. A. baumannii A118 behaved as non-motile but included the cSUA BABCDE chaperone-usher pilus assembly operon and produced biofilms on polystyrene and glass surfaces. While a known capsular polysaccharide $(K)$ locus was identified, the outer core polysaccharide $(\mathrm{OC})$ locus, which belongs to group $\mathrm{B}$, showed differences with available sequences. Our results show that despite being susceptible to most antibiotics, strain A118 conserves known virulence-related traits enhancing its value as model to study $A$. baumannii pathogenicity.

Keywords: Acinetobacter, virulence factors, pathogenicity, hospital infection, community infection, ESKAPE

\section{INTRODUCTION}

Acinetobacter baumannii infections used to be rare a few decades ago (Hartstein et al., 1988). However, its importance as an opportunistic human pathogen kept increasing and it is now responsible for a growing number of community and nosocomial infections including bacteremia, urinary tract infections, wound infections, meningitis, and pneumonia (Maragakis and Perl, 2008; Zurawski et al., 2012; McConnell et al., 2013; Harding et al., 2018). It mainly affects compromised 
patients but it has also been identified as an important causative agent of infections in wounded military personnel (Peleg et al., 2008; Petersen et al., 2011; Arivett et al., 2016). The success as an opportunistic pathogen, especially in hospital environments is due to its ability to resist desiccation and persist in the most diverse hospital locations combined with a growing resistance to disinfectants and major antibacterials (Jawad et al., 1998; Dijkshoorn et al., 2007; Perez et al., 2007; Peleg et al., 2008; Rodriguez-Bano and Bonomo, 2008; Farrow et al., 2018). Furthermore, the problematic nature of $A$. baumannii infections is enhanced by its acquisition of resistance to carbapenems, which now exceed $90 \%$ in some geographical regions with a mortality rate of about $60 \%$ (Isler et al., 2018). Recent studies identified pathogenic and resistance islands (Fournier et al., 2006; Smith et al., 2007; Post et al., 2010; Krizova et al., 2011) as well as several potential virulence factors (Harding et al., 2018) such as iron and other micronutrients uptake (Zimbler et al., 2009; Gaddy et al., 2012; Moore et al., 2014; Penwell et al., 2015), motility (Mussi et al., 2010; Eijkelkamp et al., 2013; Wood et al., 2018), production of cytotoxic and protection factors (Russo et al., 2010; Jin et al., 2011), and adhesion and biofilm formation on abiotic and biotic surfaces (Gaddy et al., 2009; Longo et al., 2014).

We have described A. baumannii A118, a naturally competent isolate obtained from blood of an infected patient, that is susceptible to numerous antibiotics including those commonly used in molecular genetics (Ramirez et al., 2010, 2011; Traglia et al., 2014). We proposed that these characteristics make this strain a convenient experimental model studying the pathobiology of this relevant human pathogen. Accordingly, A. baumannii A118 has been used in numerous studies to assess the efficiency of metal/ionophore complexes to override aminoglycoside resistance (Lin et al., 2014), to understand the effect of serum albumin in competency stability (Traglia et al., 2016; Quinn et al., 2018) and acquisition of mobile genetic elements (Ramirez et al., 2012; Domingues et al., 2018), the role of osmolarity in uptake of exogenous DNA (Domingues et al., 2019), and in the discovery of a novel bacteriophage (Turner et al., 2016). The growing number of research groups utilizing $A$. baumannii A118 as model makes characterizing this strain in greater detail desirable. In this work we focus our analysis in characteristics associated to Acinetobacter pathogenicity and virulence.

\section{MATERIALS AND METHODS}

\section{Bacterial Strains, Genomes, and Culture Conditions}

Acinetobacter baumannii A118 was isolated from the bloodstream of an infected patient in an intensive care unit (Merkier and Centron, 2006; Ramirez et al., 2010). Escherichia coli TOP10 (Invitrogen, San Diego, CA, United States) was used as host in DNA recombinant cloning. A. baumannii ATCC 17978, ATCC $19606^{\mathrm{T}}$ (Bouvet and Grimont, 1986) and the isogenic ATCC $19606^{\mathrm{T}} \mathrm{s} 1\left(\mathrm{BasD}^{-}\right)$and t6 $\left(\mathrm{BauA}^{-}\right)$ derivatives (Dorsey et al., 2004) were used in bioassays to test acinetobactin production and utilization, respectively. Iron-rich and iron-limiting conditions were attained by supplementing the growth media with $100 \mu \mathrm{M} \mathrm{FeCl}_{3}$ or $100 \mu \mathrm{M} 2,2^{\prime}$-dipyridyl (DIP), respectively.

The presence or absence of specific coding regions in the genome sequence of A. baumannii A118 (GenBank Accession Number AEOW00000000) (Ramirez et al., 2011) was determined using BLAST (Altschul et al., 1990). BLASTp searches were performed with A minimum value of $30 \%$ amino acid identity, $70 \%$ coverage and a minimum $e$-value of $1 \times 10^{-5}$. The reference genomes for sequence comparison were ATCC 17978 (Accession Number CP000521), ATCC 19606 (Accession Number ACQB00000000.1), and ACICU (Accession Number NC 010611) (Supplementary Table 1). For K Locus and OC Locus sequence analysis, we used the most related genetic structures in GenBank Database (Supplementary Table 1).

\section{Production and Utilization of Acinetobactin}

Bioassays to test production or utilization of siderophore were carried out as previously (Dorsey et al., 2004). Briefly, supernatants from cultures using succinate medium were sterilized by filtration and spotted on filter disks placed on L agar plates containing $225 \mu \mathrm{M}$ DIP seeded with the ATCC $19606^{\mathrm{T}}$ t6 strain, which does not produce the acinetobactin receptor protein BauA. The plates were incubated for $24 \mathrm{~h}$ at $37^{\circ} \mathrm{C}$, growth halos around the filters were an indication of production of a siderophore different from acinetobactin. The presence of the outer membrane protein BauA in A. baumannii strains was determined by western blotting with anti-BauA polyclonal antiserum using total lysates of bacterial cells cultured under iron-limiting or iron-rich conditions as described before (Dorsey et al., 2004). Briefly, total proteins from cells cultured under iron-rich or iron-limiting conditions were separated by SDS-PAGE using $12.5 \%$ polyacrylamide gels, transferred to a nitrocellulose membrane, and incubated in the presence of anti-BauA serum. The immunocomplexes were detected by chemiluminescence using HRP-labeled protein A (Dorsey et al., 2004).

\section{Cell Motility and Biofilm Assays}

Cell motility was assessed using semi-solid plates containing $0.3 \%$ agarose as described before (Mussi et al., 2010). The plates were inoculated on the surface with bacteria using flat-ended sterile wooden sticks or depositing $0.003 \mathrm{ml}$ of LB cultures grown to an $\mathrm{OD}_{600}$ of 0.3 . Plates were incubated for $24 \mathrm{~h}$ at $24^{\circ} \mathrm{C}$ or $37^{\circ} \mathrm{C}$ in the dark or under blue light (emission peak centered at $462 \mathrm{~nm}$ ) emitted by nine-LED (light-emitting diode) arrays with an intensity of 10 to $20 \mu \mathrm{mol}$ photons $/ \mathrm{m}^{2} / \mathrm{s}$. Biofilms formed on the walls of polystyrene or glass tubes were stained with crystal violet, visually inspected and quantified after elution of the stain as previously described (Tomaras et al., 2003). The amount of biofilm formed by each sample was normalized to its total biomass, which was determined by measuring the $\mathrm{OD}_{600}$ of duplicate cultures as described before (Tomaras et al., 2003). Triplicate assays were done at least three times using fresh samples each time. 


\section{Infection Assays}

Randomly chosen Galleria mellonella larvae were injected with $1 \times 10^{5}$ A. baumannii cells resuspended in sterile phosphatebuffered saline (PBS), or with PBS as negative control. After injection, the larvae were incubated at $37^{\circ} \mathrm{C}$ in the dark, and killing was assessed at 24-h intervals over 6 days. Caterpillars were considered dead and removed from the study if they displayed no response to probing. The results of the trial were omitted if more than two deaths occurred in the control groups. The experiments were repeated six times using 10 larvae per experimental group, and the resulting survival curves were plotted using the Kaplan-Meier method. $P$-values $<0.05$ are considered statistically significant.

\section{RESULTS AND DISCUSSION}

Acinetobacter baumannii A118 is characterized for its susceptibility to a variety of antimicrobial agents such as ceftazidime, cefepime, piperacillin, minocycline, amikacin, gentamicin, sulfamethoxazole-trimethoprim, imipenem, meropenem, and ciprofloxacin (Ramirez et al., 2010). This characteristic makes it more suitable as a model for genetic analysis than most other isolates that exhibit multiresistance to antibiotics usually used for selection in a variety of techniques. To facilitate its use as model of infection we identify the presence of genes and functions previously associated with $A$. baumannii pathogenicity. The results discussed in this article are summarized in Table 1.

\section{Iron Uptake Systems (IUSs)}

A non-specific defense system of vertebrate animals against bacterial infection is the chelation of iron by high-affinity ironbinding proteins such as transferrin and lactoferrin (Crosa and Walsh, 2002). Consequently, bacteria have evolved very efficient iron-acquisition systems to scavenge iron from the iron-binding proteins of their hosts. These systems are varied and include synthesis of a siderophore (a high-affinity ferric iron chelator) that uptakes iron and mediates its internalization

TABLE 1 | Characteristics of $A$. baumannii strains.

\begin{tabular}{|c|c|c|c|}
\hline & \multicolumn{3}{|c|}{ A. baumannii } \\
\hline & A118 & ATCC $19606^{\top}$ & ATCC 17978 \\
\hline IUS - Acinetobactin & + & + & + \\
\hline IUS - Baumannoferrin & + & - & + \\
\hline IUS - Fimsbactin & - & - & + \\
\hline Heme cluster 1 & + & + & + \\
\hline Heme cluster 2 & - & - & - \\
\hline IUS - FeoABC & + & + & + \\
\hline Biofilm & + & - & + \\
\hline Motility & + & - & + \\
\hline Capsular polysaccharide - locus $\mathrm{K}^{1}$ & PSgc8 & PSgc9 & PSgc9 \\
\hline Capsular polysaccharide - locus OC² & New & OCL1 & OCL2 \\
\hline
\end{tabular}

Plus signs denote functional systems. ${ }^{1}$ According to the classification described in Hu et al. (2013). ${ }^{2}$ According to the classification described in Kenyon et al. (2014). through a specific energy-dependent transport system (Crosa and Walsh, 2002; Di Lorenzo and Stork, 2014; Li and Ma, 2017), production of outer membrane receptors that recognize lactoferrin, transferrin, heme or non-indigenous siderophores (Koster, 2005; Antunes et al., 2011; Di Lorenzo and Stork, 2014; Huang and Wilks, 2017), or direct binding and transport of ferrous iron (Lau et al., 2016). The siderophore-mediated iron uptake systems identified in A. baumannii strains are those that utilize acinetobactin (Echenique et al., 1992; Actis et al., 1993, 1999), baumannoferrin (Penwell et al., 2015), or fimsbactins, which are represented by a family of six related chatechol/hydroxamate compounds (Proschak et al., 2013). Since the acinetobactin system, originally described in the ATCC $19606^{\mathrm{T}}$ strain (Dorsey et al., 2004; Mihara et al., 2004), is the most widespread among A. baumannii isolates we identified the presence of the gene cluster as well as entA and $e n t B$ orthologs, which code for functions required for the biosynthesis of the acinetobactin precursor 2,3-dihydroxybenzoic acid (Penwell et al., 2012). We also carried out assays to identify the regulated expression of $\mathrm{BauA}$, the outer membrane receptor of ferric-acinetobactin complexes (Figure 1A), as well as the growth of A. baumannii A118 at increasing concentrations of DIP (Figure 1B). The levels and iron regulation of BauA in A. baumannii A118 were similar to those in the control strains ATCC $19606^{\mathrm{T}}$. Both strains showed higher production of this protein as compared to the levels detected in strain ATCC 17978 (Figure 1A). However, comparison of growth of all three strains showed that while the $\mathrm{OD}_{600}$ levels reached by strains A118 and ATCC 17978 at increasing concentrations of DIP were nearly identical, strain ATCC $19606^{\mathrm{T}}$ proved to be more sensitive to iron chelation, the growth of which was completely inhibited at a lower DIP concentration when compared with the two other tested strains (Figure 1B). The A. baumannii ATCC $19606^{\mathrm{T}} \mathrm{s} 1$ mutant, deficient in production of acinetobactin due to lack of the biosynthetic enzyme BasD was inhibited at the lowest DIP concentration of all four strains tested (Figure 1B). The ability of the strains A118 and ATCC 17978 to grow at higher DIP concentrations when compared to strain ATCC $19606^{\mathrm{T}}$ could be due to the presence/expression of one or more additional iron acquisition systems or to a higher production of acinetobactin. It is known that A. baumannii ATCC $19606^{\mathrm{T}}$ does not possess the genes coding for a fimsbactin siderophore and cannot express a functional baumanoferrin iron uptake system in spite of including the cognate gene cluster. This is supported by the observation that an ATCC $19606^{\mathrm{T}}$ acinetobactin biosynthetic mutant cannot grow in the presence of DIP (Dorsey et al., 2004; Penwell et al., 2012, 2015). In addition to the acinetobactin gene cluster, A. baumannii ATCC 17978 includes the cluster coding for fimsbactin and baumannoferrin iron-uptake systems (Antunes et al., 2011; Eijkelkamp et al., 2011). Thus, the higher iron proficiency can be explained by the expression of one or both of these systems. Analysis of the A. baumannii A118 genome showed that it possesses the baumannoferrin gene cluster but not that one coding for a fimsbactin siderophore iron uptake system. To determine if besides the acinetobactin system, A. baumannii A118 expresses an additional iron uptake system we conducted a siderophore-utilization bioassays using the ATCC $19606^{\mathrm{T}}$ 


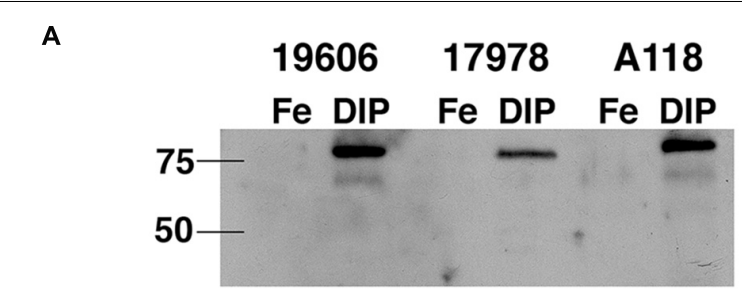

B

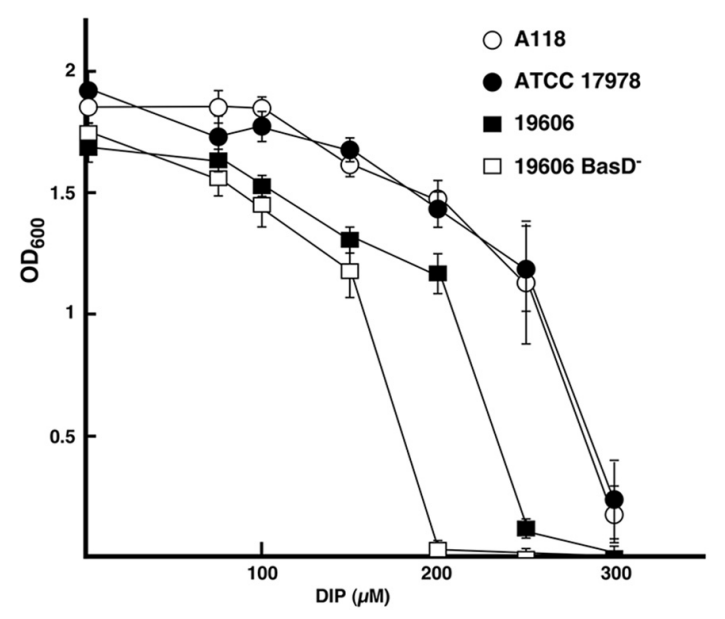

FIGURE 1 | Acinetobactin iron uptake system. (A) Synthesis and regulation of BauA. Total proteins from A. baumannii ATCC $19606^{\top}$, ATCC 17978, and A118 cultured under iron-rich $\left(100 \mu \mathrm{M} \mathrm{FeCl}_{3}\right)$ and iron-limiting (100 $\left.\mu \mathrm{M} \mathrm{DIP}\right)$ conditions were separated on a $12.5 \%$ SDS-PAGE, transferred onto nitrocellulose, and probed with anti-BauA serum. The "Precision" molecular-mass protein markers (Bio-Rad) were detected with StrepTactin-HRP conjugate. The positions of the 50- and $75-\mathrm{kDa}$ protein markers are shown on the left side to the gel. 19606, ATCC $19606^{\top} ; 17978$, ATCC 17978; Fe, iron-rich conditions; DIP, iron-limiting conditions.

(B) Bacterial growth under iron limiting conditions. The strains were cultured overnight at $37^{\circ} \mathrm{C}$ in $\mathrm{L}$ broth containing increasing concentrations of the iron chelator DIP.

t6 BauA-deficient strain, which cannot use acinetobactin to grow under iron-chelated conditions. Table 2 shows that the A. baumannii ATCC $19606^{\mathrm{T}}$ t6 BauA-deficient indicator strain was able to grow around the disks spotted with supernatants obtained from cultures of strains ATCC 17978 and A118 under iron-limiting conditions. These results strongly suggest that A. baumannii A118 secretes an additional siderophore that, given the presence the appropriate gene cluster, it most probably is baumannoferrin. However, further chemical assays are needed to prove that A118 produces baumanoferrin.

A search for genetic determinants of a heme-acquisition system showed that A. baumannii A118 posseses the heme uptake cluster 2 (Antunes et al., 2011). There is at least one report proposing that heme contributes to overcoming the iron limitation caused by the host's high affinity iron binding proteins (de Leseleuc et al., 2014). A. baumannii A118 also includes the $f e o A B C$ genes, which code for the main bacterial Feo ferrous uptake system (Cartron et al., 2006). The cluster specifies three proteins, FeoA of unknown function, the repressor FeoC, and FeoB that participates in translocation of iron across the
TABLE 2 | Siderophore utilization bioassays.

\begin{tabular}{|c|c|}
\hline Crossfeeding sample & Halo around the filter disks $(\mathrm{mm})$ \\
\hline $\mathrm{H}_{2} \mathrm{O}$ & 0 \\
\hline $\mathrm{FeCl}_{3}$ & $13.25 \pm 0.96$ \\
\hline ATCC $19606^{\top} \mathrm{Sp}$ & 0 \\
\hline ATCC $19606^{\top}$ s1 $\left(\mathrm{BasD}^{-}\right) \mathrm{Sp}$ & 0 \\
\hline ATCC 17978 Sp & $15.75 \pm 1.71$ \\
\hline A118 Sp & $13.75 \pm 2.22$ \\
\hline
\end{tabular}

Strains to be tested were cultured in succinate medium for $24 \mathrm{~h}$ at $37^{\circ} \mathrm{C}$. Culture supernatants (Sp), obtained by filter sterilization, were spotted onto filter disks that were placed on L agar plates containing $225 \mu \mathrm{M}$ DIP and seeded with the reporter A. baumannii ATCC $19606^{T}$ t6 strain deficient in the production of the BauA outer membrane acinetobactin receptor. Halos were measured after incubation for $24 \mathrm{~h}$ at $37^{\circ} \mathrm{C} . \mathrm{H}_{2} \mathrm{O}$ or $\mathrm{FeCl}_{3}$ were spotted as negative and positive controls, respectively.

inner membrane. A recent study showed that although the Feo system enhances growth in heat-inactivated human serum and contributes to resistance to human serum, it is not essential for virulence (Runci et al., 2019).

\section{Biofilm Formation}

The ability of $A$. baumannii strains to form biofilms on abiotic surfaces (Tomaras et al., 2003) is partially or totally responsible for their persistence in different environments such as the surface of medical devices and other common artifacts like furniture, linens, soap dispensers, phones and computer keyboards (Hartstein et al., 1988, 1990; Neely, 2000; Villegas and Hartstein, 2003; Borer et al., 2005; Espinal et al., 2012). Comparative analysis of the A. baumannii A118 nucleotide sequence with $A$. baumannii genomes showed that the $c s u A / B A B C D E$ chaperon-usher pilus assembly system is present in the A118 strain and the identities of the deduced amino acid sequence of the products of the cognate open reading frames is $96 \%$ or higher when compared to those from strain ATCC 17978. Standard crystal violet biofilm assays using polystyrene tubes showed that A. baumannii A118 formed a distinctive biofilm ring on the surface of the tubes as it was described for ATCC $19606^{\mathrm{T}}$ cells (Tomaras et al., 2003). Control assays showed a stronger ring in the case of the strain ATCC $19606^{\mathrm{T}}$ while significantly reduced biofilm was formed by strain ATCC 17978. Quantification of total biofilms formed on polystyrene and glass showed that strain A118 produced a significant amount of biofilm on glass and polystyrene surfaces (Figure 2). It was of interest that while the biofilm mass was higher for strain A118 than for strain ATCC $19606^{\mathrm{T}}$ on glass, the opposite was true for biofilm formed on plastic (Figure 2). Although the molecular and cellular bases of these differences are unknown, variations in the amount of biofilms formed by different strains on the same or different types of surfaces seem to be a common property among A. baumannii clinical isolates (McQueary and Actis, 2011; Runci et al., 2017; Quinn et al., 2018; Wood et al., 2018).

\section{Motility}

Acinetobacter baumannii lacks flagellum and therefore cannot show flagellum-mediated swimming motility (Towner et al., 1991). However, it has been shown that Acinetobacter strains 


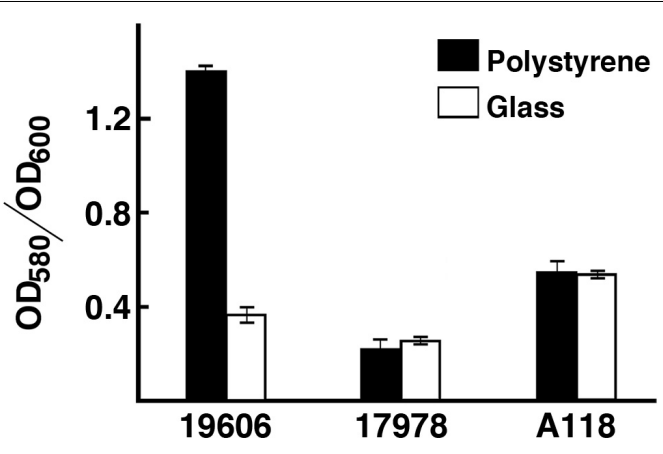

FIGURE 2 | Production of biofilm. Quantification of biofilm formation on polystyrene and glass tubes by $A$. baumannii strains ATCC $19606^{\top}$, ATCC 17978 , and $\mathrm{A} 118$ after static incubation at $37^{\circ} \mathrm{C}$. Quantification was carried out as described in "Materials and Methods."

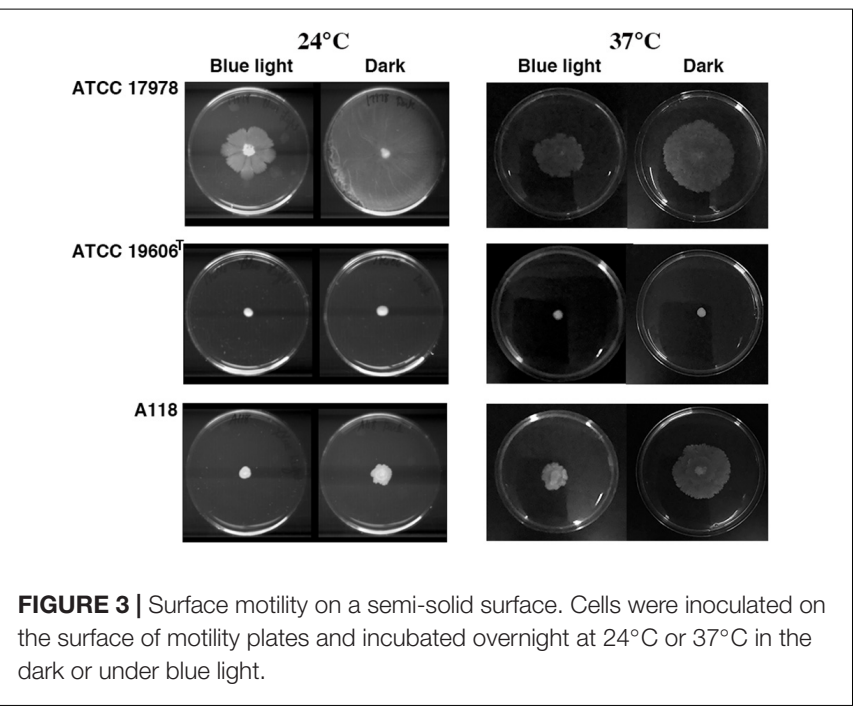

are capable of motility on semi-solid agar (Barker and Maxted, 1975; Mussi et al., 2010; Eijkelkamp et al., 2011; Quinn et al., 2018; Wood et al., 2018), and the movement is dependent on the concentration of iron in the environment and possibly on other stress related factors (Eijkelkamp et al., 2011; Quinn et al., 2018; Wood et al., 2018). Furthermore, it has been shown that at $24^{\circ} \mathrm{C}$ strain ATCC 17978 shows low or no signs of motility when growing exposed to light but this situation is reversed when growing in the dark (Mussi et al., 2010; Figure 3). This regulation depends on the expression of BlsA, a photoreceptor and transcriptional regulator (Mussi et al., 2010), and the newly identified PrpABCD type I pilus assembly system (Wood et al., 2018). In contrast, as it was shown before, ATCC 17978 cells displayed comparable surface motility independently of illumination when cultured at $37^{\circ} \mathrm{C}$ (Mussi et al., 2010). Analysis of A. baumannii A118 showed that this strain was unable to exhibit motility under blue light at $24^{\circ} \mathrm{C}$ but it showed slight motility in the dark (Figure 3). This motility was more evident at $37^{\circ} \mathrm{C}$ in the dark although cells displayed a reduced motility when cultured at this temperature under illumination (Figure 3). A. baumannii ATCC $19606^{\mathrm{T}}$ did not show motility in any of the assayed conditions (Figure 3). Analysis of the A. baumannii A118 genome showed the presence of all the genes associated with a type IV pilus system (T4P), known to play a role in A. baumannii twitching motility and surface-associated. Taken together, these results indicate that A. baumannii A118 is motile under the right conditions and that light and temperature affect the motility responses of different $A$. baumannii strains by mechanisms that remain to characterized.

\section{Locus $\mathrm{K}$ and Locus $\mathrm{OC}$}

Acinetobacter baumannii strains produce a capsular polysaccharide coded for by a gene cluster known as K locus, which exhibits sequence variations among isolates (Kenyon and Hall, 2013). Another surface polysaccharide produced by this bacterium is part of the lipooligosaccharide formed by the lipid A and the inner and outer core oligosaccharides (Kenyon et al., 2014). While the inner core oligosaccharide is well conserved, the outer core is highly variable (Erridge et al., 2007). The group of genes

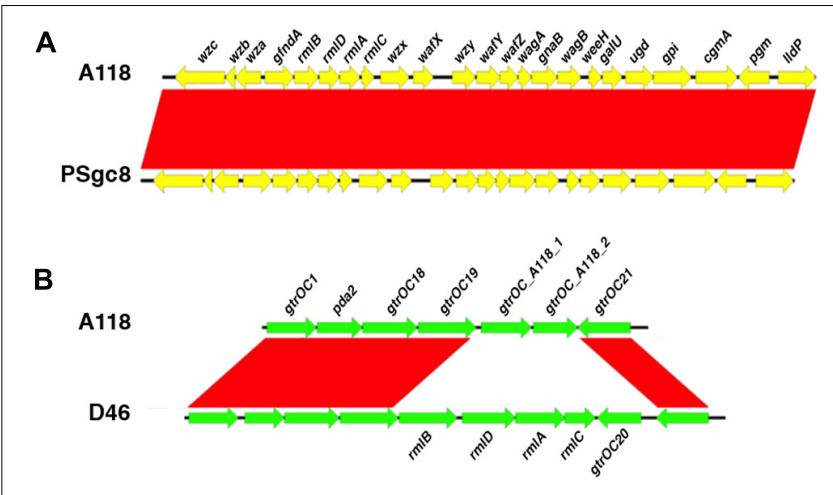

FIGURE 4 | $\mathrm{K}$ and OC loci comparisons. (A) Comparison of the $\mathrm{K}$ loci from A. baumannii strains A118 and PSgc8 (Accession Number LUH5538). Comparison was performed using BLAST (version 2.2.31) and the graphic representation was generated using the software EasyFig (version 2.1). Proteins coded for by the genes are: wzc, Tyrosine-protein kinase; wzb, protein-tyrosine-phosphatase; wza, periplasmic protein; gnaA, UDP-glucose/GDP-mannose dehydrogenase; $r m / B$, dTDP-D-glucose-4,6-dehydratase; rmID, dTDP-4-dehydrorhamnose reductase; $r m / A$, dTDP-glucose pyrophosphorylase; $r m / C$,

dTDP-4-keto-6-deoxy-D-glucose-3,5-epimerase; wzX, flippase; waf $X$, glycosyltransferase; wzy, O antigen polymerase; waf $Y$, glycosyltransferase; wafZ, glycosyltransferase; wagA, acetyltransferase; gnaB, NAD-dependent epimerase/dehydratase; $w a g B$, glycosyl transferase; $w e e H$,

glycosyltransferase; galU, UDP-glucose pyrophosphorylase; ugd,

UDP-glucose 6-dehydrogenase; gpi, glucose-6-phosphate isomerase; cgmA, cyclic beta-1,2-glucan modification transmembrane protein; pgm, phosphoglucomutase; IldP, L-lactate permease. (B) Comparison of the OC loci from A. baumannii strains A118 and D46 (Accession Number KF030679). All three strains, D46, ZW85-1 (Accession Number CP006768), and 6200 (Accession Number CP010397), have identical OC loci. Proteins coded for by the genes are: gtrOC1, glycosyltransferase; pda2, polysaccharide deacetylase; gtrOC18, glycosyltransferase; gtrOC19, glycosyltransferase; gtrOC20, glycosyltransferase; gtrOC21, glycosyltransferase; gtrOC_A118_1, capsular protein, polysaccharide synthesis; gtrOC_A118_2, glycosyltransferase. 


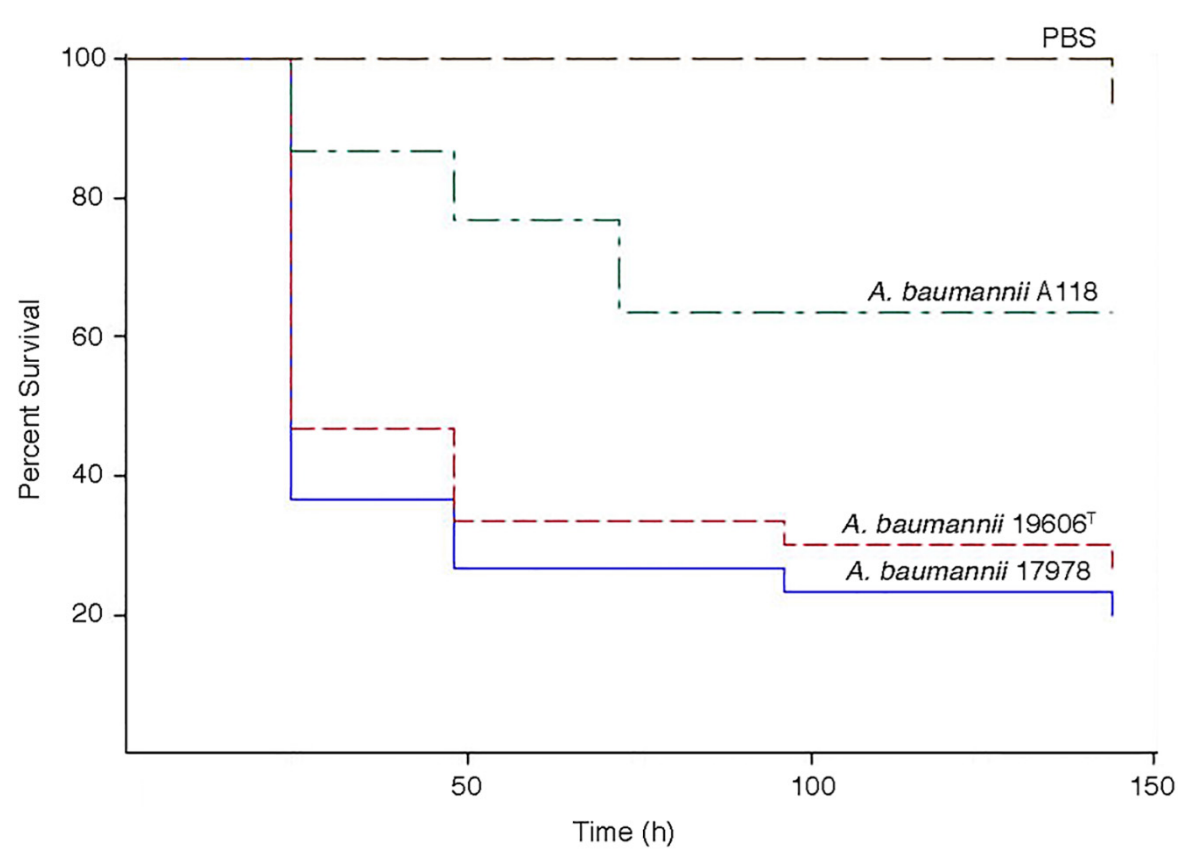

FIGURE 5 | Galleria mellonella infection assays. Infections were carried out as described in Materials and Methods. Larvae were injected with $1 \times 10^{5}$ cells of A. baumannii $\mathrm{A} 118,19606^{\top}$, or 17978 resuspended in sterile PBS. Negative controls were injected with PBS.

coding for the outer core oligosaccharide are known as the OC locus. The surface carbohydrates have been linked to the virulence of $A$. baumannii in numerous studies (Russo et al., 2010; Iwashkiw et al., 2012; Geisinger and Isberg, 2015). We found the A. baumannii A118 K locus structure located between the $f k p A$ and $l l d P$ genes as it has been reported for other strains (Kenyon and Hall, 2013). Nucleotide sequence comparison of this locus showed $99 \%$ identity (100\% cover) with that of the strain Sv8/PSgc8 described by Hu et al. (2013); (Figure 4A). A search to identify the OC locus showed that an A. baumannii A118 gene cluster exhibited $98 \%$ identity ( $79 \%$ cover) with those present in strains D46, ZW85-1, and 6200 (Figure 4B). None of the reported versions of the OC locus was identical to that of the A. baumannii A118 strain. The two genes not represented in strain D46 show $100 \%$ amino acid sequence identity with those found in strain Naval-72. On the other hand, the $r m l B, r m l D, r m l A$ y $r m l C$ genes present in this locus in strain D46 are located in the $\mathrm{K}$ locus in A. baumannii A118 (see Figures 4A,B). According to the OC loci classification proposed by Kenyon et al. (2014), the A. baumannii A118 OC locus belongs to group B.

\section{Experimental Infections}

The virulence of A. baumannii A118 was assessed using the G. mellonella model of infection. Figure 5 shows that larvae infections with this strain results in a significant increase in mortality as compared to the negative control. However, strain A118 showed lower virulence when compared to the A. baumannii ATCC 17978 and ATCC 19606T strains, both of which produced comparable larvae survival rates (Figure 5).

\section{CONCLUDING REMARKS}

There is an urgent need to find answers to the problem of infections with bacteria belonging to the ESKAPE group (Boucher et al., 2013), which includes A. baumannii. Research on this bacterium presents numerous challenges due to its tremendous genetic plasticity and diversity, as well as its multiresistance to most antibiotics used for selection in the molecular biology laboratory (Ramirez et al., 2010; Wright et al., 2016; Traglia et al., 2019). We have analyzed functions related to the antibiotic resistance/susceptibility profile of the model strain A. baumannii A118 (Ramirez et al., 2010, 2011). Building on the characterization of this strain we show here the presence of genetic and cellular traits that could be associated to its pathogenicity. It most probably includes at least two iron uptake systems, those mediated by the siderophores acinetobactin and baumannoferrin (Dorsey et al., 2003, 2004; Mihara et al., 2004; Eijkelkamp et al., 2011). The presence of the $c s u$ operon is compatible with the ability of A. baumannii A118 to form biofilms on polystyrene and glass to levels comparable to strain ATCC $19606^{\mathrm{T}}$. The $\mathrm{K}$ and OC loci were identified and characterized, while the $\mathrm{K}$ locus has been found in another isolated, the OC locus was still undescribed. Although A. baumannii A118 displayed more surface motility than ATCC $19606^{\mathrm{T}}$ under all tested conditions, its motility seems reduced when compared to ATCC17978, particularly under illumination at $37^{\circ} \mathrm{C}$. This response could be due to the absence of the gene coding for a fimbrial protein that might be involved in this response. In conclusion, A. baumannii A118 possesses the main potential virulence factors found in other clinical isolates 
reinforcing the usefulness of this strain as a model of genetic manipulation and pathogenic studies.

\section{DATA AVAILABILITY}

All datasets generated for this study are included in the manuscript and/or the Supplementary Files.

\section{AUTHOR CONTRIBUTIONS}

MT, MR, LA, and RB conceived and designed the experiments. MR, WP, JG, GT, DZ, NN, BA, MA, LA, and MT performed the experiments. MR, WP, JG, NN, GT, DZ, BA, MA, LA, and MT analyzed the data. MA, MR, MT, and NN contributed reagents, mutant strains, materials, and analysis tools. $\mathrm{MR}$ and MT wrote the manuscript. MR, MT, LA, RB, and NN revised the manuscript.

\section{REFERENCES}

Actis, L. A., Smoot, J. C., Barancin, C. E., and Findlay, R. H. (1999). Comparison of differential plating media and two chromatography techniques for the detection of histamine production in bacteria. J. Microbiol. Methods 39, 79-90. doi: 10.1016/s0167-7012(99)00099-8

Actis, L. A., Tolmasky, M. E., Crosa, L. M., and Crosa, J. H. (1993). Effect of ironlimiting conditions on growth of clinical isolates of Acinetobacter baumannii. J. Clin. Microbiol. 31, 2812-2815.

Altschul, S. F., Gish, W., Miller, W., Myers, E. W., and Lipman, D. J. (1990). Basic local alignment search tool. J. Mol. Biol. 215, 403-410. doi: 10.1006/jmbi.1990. 9999

Antunes, L. C., Imperi, F., Towner, K. J., and Visca, P. (2011). Genome-assisted identification of putative iron-utilization genes in Acinetobacter baumannii and their distribution among a genotypically diverse collection of clinical isolates. Res. Microbiol. 162, 279-284. doi: 10.1016/j.resmic.2010.10.010

Arivett, B. A., Ream, D. C., Fiester, S. E., Kidane, D., and Actis, L. A. (2016). Draft genome sequences of Acinetobacter baumannii isolates from wounded military personnel. Genome Announc. 4:e773-16. doi: 10.1128/genomeA. 00773-16

Barker, J., and Maxted, H. (1975). Observations on the growth and movement of Acinetobacter on semi-solid media. J. Med. Microbiol. 8, 443-446. doi: 10.1099/00222615-8-3-443

Borer, A., Gilad, J., Smolyakov, R., Eskira, S., Peled, N., Porat, N., et al. (2005). Cell phones and Acinetobacter transmission. Emerg. Infect. Dis. 11, 1160-1161.

Boucher, H. W., Talbot, G. H., Benjamin, D. K. Jr., Bradley, J., Guidos, R. J., Jones, R. N., et al. (2013). 10 x'20 Progress-development of new drugs active against gram-negative bacilli: an update from the infectious diseases society of America. Clin. Infect. Dis. 56, 1685-1694. doi: 10.1093/cid/cit152

Bouvet, P. J. M., and Grimont, P. A. D. (1986). Taxonomy of the genus Acinetobacter with the recognition of Acinetobacter baumannii sp. nov., Acinetobacter haemolyticus sp. nov., Acinetobacter johnsonii sp. nov., and Acinetobacter junii sp. nov. and emended descriptions of Acinetobacter calcoaceticus and Acinetobacter lwoffii. Int. J. Syst. Bacteriol. 36, 228-240. doi: 10.1099/00207713-36-2-228

Cartron, M. L., Maddocks, S., Gillingham, P., Craven, C. J., and Andrews, S. C. (2006). Feo-transport of ferrous iron into bacteria. Biometals 19, 143-157. doi: 10.1007/s10534-006-0003-2

Crosa, J. H., and Walsh, C. T. (2002). Genetics and assembly line enzymology of siderophore biosynthesis in bacteria. Microbiol. Mol. Biol. Rev. 66, 223-249. doi: $10.1128 / \mathrm{mmbr}$.66.2.223-249.2002

de Leseleuc, L., Harris, G., Kuolee, R., Xu, H. H., and Chen, W. (2014). Serum resistance, gallium nitrate tolerance and extrapulmonary dissemination

\section{FUNDING}

This study was supported by Public Health Service grants 2R15AI047115 (to MT), AI070174 (to LA), R01AI00560, R01AI0AI063517, R21AI11458, and R01AI072219 (to RB), and SC3G-M125556 (to MR) from the National Institutes of Health, award 1I01BX001974 (to RB) from the BLRDS (Veterans Administration Office of Research and Development), and award VISN10 (to RB) from the Geriatric Research and Educational and Clinical Center. The funders had no role in study design, data collection and analysis, decision to publish, or preparation of the manuscript.

\section{SUPPLEMENTARY MATERIAL}

The Supplementary Material for this article can be found online at: https://www.frontiersin.org/articles/10.3389/fmicb. 2019.01599/full\#supplementary-material

are linked to heme consumption in a bacteremic strain of Acinetobacter baumannii. Int. J. Med. Microbiol. 304, 360-369. doi: 10.1016/j.ijmm.2013. 12.002

Di Lorenzo, M., and Stork, M. (2014). Plasmid-encoded iron uptake systems. Microbiol. Spectr. 2:6.

Dijkshoorn, L., Nemec, A., and Seifert, H. (2007). An increasing threat in hospitals: multidrug-resistant Acinetobacter baumannii. Nat. Rev. Microbiol. 5, 939-951. doi: 10.1038/nrmicro1789

Domingues, S., Rosario, N., Ben Cheikh, H., and Da Silva, G. J. (2018). ISAba1 and Tn6168 acquisition by natural transformation leads to third-generation cephalosporins resistance in Acinetobacter baumannii. Infect. Genet. Evol. 63, 13-16. doi: 10.1016/j.meegid.2018.05.007

Domingues, S., Rosario, N., Candido, A., Neto, D., Nielsen, K. M., and Da Silva, G. J. (2019). Competence for natural transformation is common among clinical strains of resistant Acinetobacter spp. Microorganisms 7:30. doi: 10 . 3390/microorganisms7020030

Dorsey, C. W., Tolmasky, M. E., Crosa, J. H., and Actis, L. A. (2003). Genetic organization of an Acinetobacter baumannii chromosomal region harbouring genes related to siderophore biosynthesis and transport. Microbiol. SGM 149, 1227-1238. doi: 10.1099/mic.0.26204-0

Dorsey, C. W., Tomaras, A. P., Connerly, P. L., Tolmasky, M. E., Crosa, J. H., and Actis, L. A. (2004). The siderophore-mediated iron acquisition systems of Acinetobacter baumannii ATCC 19606 and Vibrio anguillarum 775 are structurally and functionally related. Microbiology 150, 3657-3667. doi: 10 . 1099/mic.0.27371-0

Echenique, J. R., Arienti, H., Tolmasky, M. E., Read, R. R., Staneloni, R. J., Crosa, J. H., et al. (1992). Characterization of a high-affinity iron transport system in Acinetobacter baumannii. J. Bacteriol. 174, 7670-7679. doi: 10.1128/jb.174.23. 7670-7679.1992

Eijkelkamp, B. A., Hassan, K. A., Paulsen, I. T., and Brown, M. H. (2011). Investigation of the human pathogen Acinetobacter baumannii under iron limiting conditions. BMC Genomics 12:126. doi: 10.1186/1471-2164-12-126

Eijkelkamp, B. A., Stroeher, U. H., Hassan, K. A., Elbourne, L. D., Paulsen, I. T., and Brown, M. H. (2013). H-NS plays a role in expression of Acinetobacter baumannii virulence features. Infect. Immun. 81, 2574-2583. doi: 10.1128/IAI. 00065-13

Erridge, C., Moncayo-Nieto, O. L., Morgan, R., Young, M., and Poxton, I. R. (2007). Acinetobacter baumannii lipopolysaccharides are potent stimulators of human monocyte activation via toll-like receptor 4 signalling. J. Med. Microbiol. 56, 165-171. doi: 10.1099/jmm.0.46823-0

Espinal, P., Marti, S., and Vila, J. (2012). Effect of biofilm formation on the survival of Acinetobacter baumannii on dry surfaces. J. Hosp. Infect. 80, 56-60. doi: 10.1016/j.jhin.2011.08.013 
Farrow, J. M. III, Wells, G., and Pesci, E. C. (2018). Desiccation tolerance in Acinetobacter baumannii is mediated by the two-component response regulator BfmR. PLoS One 13:e0205638. doi: 10.1371/journal.pone.0205638

Fournier, P. E., Vallenet, D., Barbe, V., Audic, S., Ogata, H., Poirel, L., et al. (2006). Comparative genomics of multidrug resistance in Acinetobacter baumannii. PLoS Genet. 2:e7. doi: 10.1371/journal.pgen.0020007

Gaddy, J. A., Arivett, B. A., Mcconnell, M. J., Lopez-Rojas, R., Pachon, J., and Actis, L. A. (2012). Role of acinetobactin-mediated iron acquisition functions in the interaction of Acinetobacter baumannii strain ATCC 19606T with human lung epithelial cells, Galleria mellonella caterpillars, and mice. Infect. Immun. 80, 1015-1024. doi: 10.1128/IAI.06279-11

Gaddy, J. A., Tomaras, A. P., and Actis, L. A. (2009). The Acinetobacter baumannii 19606 OmpA protein plays a role in biofilm formation on abiotic surfaces and in the interaction of this pathogen with eukaryotic cells. Infect. Immun. 77, 3150-3160. doi: 10.1128/IAI.00096-09

Geisinger, E., and Isberg, R. R. (2015). Antibiotic modulation of capsular exopolysaccharide and virulence in Acinetobacter baumannii. PLoS Pathog. 11:e1004691. doi: 10.1371/journal.ppat.1004691

Harding, C. M., Hennon, S. W., and Feldman, M. F. (2018). Uncovering the mechanisms of Acinetobacter baumannii virulence. Nat. Rev. Microbiol. 16, 91-102. doi: 10.1038/nrmicro.2017.148

Hartstein, A. I., Morthland, V. H., Rourke, J. W. Jr., Freeman, J., Garber, S., Sykes, R., et al. (1990). Plasmid DNA fingerprinting of Acinetobacter calcoaceticus subspecies anitratus from intubated and mechanically ventilated patients. Infect. Control Hosp. Epidemiol. 11, 531-538. doi: 10.2307/30151321

Hartstein, A. I., Rashad, A. L., Liebler, J. M., Actis, L. A., Freeman, J., Rourke, J. W., et al. (1988). Multiple intensive care unit outbreak of Acinetobacter calcoaceticus subspecies anitratus respiratory infection and colonization associated with contaminated, reusable ventilator circuits and resuscitation bags. Am. J. Med. 85, 624-631. doi: 10.1016/0002-9343(88)90683-3

Hu, D., Liu, B., Dijkshoorn, L., Wang, L., and Reeves, P. R. (2013). Diversity in the major polysaccharide antigen of Acinetobacter baumannii assessed by DNA sequencing, and development of a molecular serotyping scheme. PLoS One 8:e70329. doi: 10.1371/journal.pone.0070329

Huang, W., and Wilks, A. (2017). Extracellular heme uptake and the challenge of bacterial cell membranes. Annu. Rev. Biochem. 86, 799-823. doi: 10.1146/ annurev-biochem-060815-014214

Isler, B., Doi, Y., Bonomo, R. A., and Paterson, D. L. (2018). New treatment options against carbapenem-resistant Acinetobacter baumannii infections. Antimicrob. Agents Chemother. 63, e01110-e01118. doi: 10.1128/AAC.01110-18

Iwashkiw, J. A., Seper, A., Weber, B. S., Scott, N. E., Vinogradov, E., Stratilo, C., et al. (2012). Identification of a general O-linked protein glycosylation system in Acinetobacter baumannii and its role in virulence and biofilm formation. PLoS Pathog. 8:e1002758. doi: 10.1371/journal.ppat.1002758

Jawad, A., Seifert, H., Snelling, A. M., Heritage, J., and Hawkey, P. M. (1998). Survival of Acinetobacter baumannii on dry surfaces: comparison of outbreak and sporadic isolates. J. Clin. Microbiol. 36, 1938-1941.

Jin, J. S., Kwon, S. O., Moon, D. C., Gurung, M., Lee, J. H., Kim, S. I., et al. (2011). Acinetobacter baumannii secretes cytotoxic outer membrane protein a via outer membrane vesicles. PLoS One 6:e17027. doi: 10.1371/journal.pone. 0017027

Kenyon, J. J., and Hall, R. M. (2013). Variation in the complex carbohydrate biosynthesis loci of Acinetobacter baumannii genomes. PLoS One 8:e62160. doi: 10.1371 /journal.pone.0062160

Kenyon, J. J., Nigro, S. J., and Hall, R. M. (2014). Variation in the OC locus of Acinetobacter baumannii genomes predicts extensive structural diversity in the lipooligosaccharide. PLoS One 9:e107833. doi: 10.1371/journal.pone.0107833

Koster, W. (2005). Cytoplasmic membrane iron permease systems in the bacterial cell envelope. Front. Biosci. 10:462-477.

Krizova, L., Dijkshoorn, L., and Nemec, A. (2011). Diversity and evolution of AbaR genomic resistance islands in Acinetobacter baumannii strains of European clone I. Antimicrob. Agents Chemother. 55, 3201-3206. doi: 10.1128/AAC. 00221-11

Lau, C. K., Krewulak, K. D., and Vogel, H. J. (2016). Bacterial ferrous iron transport: the Feo system. FEMS Microbiol. Rev. 40, 273-298. doi: 10.1093/femsre/fuv049

Li, Y., and Ma, Q. (2017). Iron acquisition strategies of Vibrio anguillarum. Front. Cell Infect. Microbiol. 7:342. doi: 10.3389/fcimb.2017.00342
Lin, D. L., Tran, T., Alam, J. Y., Herron, S. R., Ramirez, M. S., and Tolmasky, M. E. (2014). Inhibition of aminoglycoside 6'-N-acetyltransferase type Ib by zinc: reversal of amikacin resistance in Acinetobacter baumannii and Escherichia coli by a zinc ionophore. Antimicrob. Agents Chemother. 58, 4238-4241. doi: 10.1128/AAC.00129-14

Longo, F., Vuotto, C., and Donelli, G. (2014). Biofilm formation in Acinetobacter baumannii. New Microbiol. 37, 119-127.

Maragakis, L. L., and Perl, T. M. (2008). Acinetobacter baumannii: epidemiology, antimicrobial resistance, and treatment options. Clin. Infect. Dis. 46, 1254-1263. doi: $10.1086 / 529198$

McConnell, M. J., Actis, L., and Pachon, J. (2013). Acinetobacter baumannii: human infections, factors contributing to pathogenesis and animal models. FEMS Microbiol. Rev. 37, 130-155. doi: 10.1111/j.1574-6976.2012.00344.x

McQueary, C. N., and Actis, L. A. (2011). Acinetobacter baumannii biofilms: variations among strains and correlations with other cell properties. J. Microbiol. 49, 243-250. doi: 10.1007/s12275-011-0343-7

Merkier, A. K., and Centron, D. (2006). bla(OXA-51)-type beta-lactamase genes are ubiquitous and vary within a strain in Acinetobacter baumannii. Int. J. Antimicrob. Agents 28, 110-113. doi: 10.1016/j.ijantimicag.2006.03.023

Mihara, K., Tanabe, T., Yamakawa, Y., Funahashi, T., Nakao, H., Narimatsu, S., et al. (2004). Identification and transcriptional organization of a gene cluster involved in biosynthesis and transport of acinetobactin, a siderophore produced by Acinetobacter baumannii ATCC 19606T. Microbiology 150, 2587-2597. doi: 10.1099/mic.0.27141-0

Moore, J. L., Becker, K. W., Nicklay, J. J., Boyd, K. L., Skaar, E. P., and Caprioli, R. M. (2014). Imaging mass spectrometry for assessing temporal proteomics: analysis of calprotectin in Acinetobacter baumannii pulmonary infection. Proteomics 14, 820-828. doi: 10.1002/pmic.201300046

Mussi, M. A., Gaddy, J. A., Cabruja, M., Arivett, B. A., Viale, A. M., Rasia, R., et al. (2010). The opportunistic human pathogen Acinetobacter baumannii senses and responds to light. J. Bacteriol. 192, 6336-6345. doi: 10.1128/JB.00917-10

Neely, A. N. (2000). A survey of gram-negative bacteria survival on hospital fabrics and plastics. J. Burn. Care Rehabil. 21, 523-527. doi: 10.1097/00004630200021060-00009

Peleg, A. Y., Seifert, H., and Paterson, D. L. (2008). Acinetobacter baumannii: emergence of a successful pathogen. Clin. Microbiol. Rev. 21, 538-582. doi: 10.1128/CMR.00058-07

Penwell, W. F., Arivett, B. A., and Actis, L. A. (2012). The Acinetobacter baumannii ent A gene located outside the acinetobactin cluster is critical for siderophore production, iron acquisition, and virulence. PLoS One 7:e36493. doi: 10.1371/ journal.pone.0036493

Penwell, W. F., Degrace, N., Tentarelli, S., Gauthier, L., Gilbert, C. M., Arivett, B. A., et al. (2015). Discovery and characterization of new hydroxamate siderophores, Baumannoferrin A and B, produced by Acinetobacter baumannii. Chembiochem 16, 1896-1904. doi: 10.1002/cbic.201500147

Perez, F., Hujer, A. M., Hujer, K. M., Decker, B. K., Rather, P. N., and Bonomo, R. A. (2007). Global challenge of multidrug-resistant Acinetobacter baumannii. Antimicrob. Agents Chemother. 51, 3471-3484.

Petersen, K., Cannegieter, S. C., Van Der Reijden, T. J., Van Strijen, B., You, D. M., Babel, B. S., et al. (2011). Diversity and clinical impact of Acinetobacter baumannii colonization and infection at a military medical center. J. Clin. Microbiol. 49, 159-166. doi: 10.1128/JCM.00766-10

Post, V., White, P. A., and Hall, R. M. (2010). Evolution of AbaR-type genomic resistance islands in multiply antibiotic-resistant Acinetobacter baumannii. J. Antimicrob. Chemother. 65, 1162-1170. doi: 10.1093/jac/dkq095

Proschak, A., Lubuta, P., Grun, P., Lohr, F., Wilharm, G., De Berardinis, V., et al. (2013). Structure and biosynthesis of fimsbactins A-F, siderophores from Acinetobacter baumannii and Acinetobacter baylyi. Chembiochem 14, 633-638. doi: 10.1002/cbic. 201200764

Quinn, B., Rodman, N., Jara, E., Fernandez, J. S., Martinez, J., Traglia, G. M., et al. (2018). Human serum albumin alters specific genes that can play a role in survival and persistence in Acinetobacter baumannii. Sci. Rep. 8:14741. doi: 10.1038/s41598-018-33072-z

Ramirez, M. S., Adams, M. D., Bonomo, R. A., Centron, D., and Tolmasky, M. E. (2011). Genomic analysis of Acinetobacter baumannii A118 by comparison of optical maps: identification of structures related to its susceptibility phenotype. Antimicrob. Agents Chemother. 55, 1520-1526. doi: 10.1128/AAC.01595-10 
Ramirez, M. S., Don, M., Merkier, A. K., Bistue, A. J., Zorreguieta, A., Centron, D., et al. (2010). Naturally competent Acinetobacter baumannii clinical isolate as a convenient model for genetic studies. J. Clin. Microbiol. 48, 1488-1490. doi: 10.1128/JCM.01264-09

Ramirez, M. S., Merkier, A. K., Quiroga, M. P., and Centron, D. (2012). Acinetobacter baumannii is able to gain and maintain a plasmid harbouring In 35 found in Enterobacteriaceae isolates from Argentina. Curr. Microbiol. 64, 211-213. doi: 10.1007/s00284-011-0052-9

Rodriguez-Bano, J., and Bonomo, R. A. (2008). Multidrug-resistant Acinetobacter baumannii: eyes wide shut? Enferm. Infecc. Microbiol. Clin. 26, 185-186. doi: 10.1016/s0213-005x(08)72688-0

Runci, F., Bonchi, C., Frangipani, E., Visaggio, D., and Visca, P. (2017). Acinetobacter baumannii biofilm formation in human serum and disruption by gallium. Antimicrob. Agents Chemother. 61:e1563-16. doi: 10.1128/AAC.01563-16

Runci, F., Gentile, V., Frangipani, E., Rampioni, G., Leoni, L., Lucidi, M., et al. (2019). Contribution of active iron-uptake to Acinetobacter baumannii pathogenicity. Infect. Immun. 87:e755-18. doi: 10.1128/IAI.00755-18

Russo, T. A., Luke, N. R., Beanan, J. M., Olson, R., Sauberan, S. L., Macdonald, U., et al. (2010). The K1 capsular polysaccharide of Acinetobacter baumannii strain 307-0294 is a major virulence factor. Infect. Immun. 78, 3993-4000. doi: 10.1128/IAI.00366-10

Smith, M. G., Gianoulis, T. A., Pukatzki, S., Mekalanos, J. J., Ornston, L. N., Gerstein, M., et al. (2007). New insights into Acinetobacter baumannii pathogenesis revealed by high-density pyrosequencing and transposon mutagenesis. Genes Dev. 21, 601-614. doi: 10.1101/gad.1510307

Tomaras, A. P., Dorsey, C. W., Edelmann, R. E., and Actis, L. A. (2003). Attachment to and biofilm formation on abiotic surfaces by Acinetobacter baumannii: involvement of a novel chaperone-usher pili assembly system. Microbiology 149, 3473-3484. doi: 10.1099/mic.0.26541-0

Towner, K. J., Bergogne-Berezin, E., and Fewson, C. A. (1991). “Acinetobacter: portrait of a genus," in The Biology of Acinetobacter, ed. C. A. Fewson (New York, NY: Plenum Press), 1-24. doi: 10.1007/978-1-48993553-3_1

Traglia, G. M., Chua, K., Centron, D., Tolmasky, M. E., and Ramirez, M. S. (2014). Whole-genome sequence analysis of the naturally competent Acinetobacter baumannii clinical isolate A118. Genome Biol. Evol. 6, 2235-2239. doi: 10.1093/ gbe/evu176

Traglia, G. M., Place, K., Dotto, C., Fernandez, J. S., Montana, S., Bahiense, C. D. S., et al. (2019). Interspecies DNA acquisition by a naturally competent
Acinetobacter baumannii strain. Int. J. Antimicrob. Agents 53, 483-490. doi: 10.1016/j.ijantimicag.2018.12.013

Traglia, G. M., Quinn, B., Schramm, S. T., Soler-Bistue, A., and Ramirez, M. S. (2016). Serum albumin and $\mathrm{Ca} 2+$ are natural competence inducers in the human pathogen Acinetobacter baumannii. Antimicrob. Agents Chemother. 60, 4920-4929. doi: 10.1128/AAC.00529-16

Turner, D., Wand, M. E., Sutton, J. M., Centron, D., Kropinski, A. M., and Reynolds, D. M. (2016). Genome sequence of vB_AbaS_TRS1, a viable prophage isolated from Acinetobacter baumannii strain A118. Genome Announc. 4:e1051-16. doi: 10.1128/genomeA.01051-16

Villegas, M. V., and Hartstein, A. I. (2003). Acinetobacter outbreaks, 1977-2000. Infect. Control. Hosp. Epidemiol. 24, 284-295. doi: 10.1086/502205

Wood, C. R., Ohneck, E. J., Edelmann, R. E., and Actis, L. A. (2018). A lightregulated type I pilus contributes to Acinetobacter baumannii biofilm, motility, and virulence functions. Infect. Immun. 86:e442-18. doi: 10.1128/IAI.00442-18

Wright, M. S., Iovleva, A., Jacobs, M. R., Bonomo, R. A., and Adams, M. D. (2016). Genome dynamics of multidrug-resistant Acinetobacter baumannii during infection and treatment. Genome Med. 8:26. doi: 10.1186/s13073-0160279-y

Zimbler, D. L., Penwell, W. F., Gaddy, J. A., Menke, S. M., Tomaras, A. P., Connerly, P. L., et al. (2009). Iron acquisition functions expressed by the human pathogen Acinetobacter baumannii. Biometals 22, 23-32. doi: 10.1007/s10534-0089202-3

Zurawski, D. V., Thompson, M. G., Mcqueary, C. N., Matalka, M. N., Sahl, J. W., Craft, D. W., et al. (2012). Genome sequences of four divergent multidrugresistant Acinetobacter baumannii strains isolated from patients with sepsis or osteomyelitis. J. Bacteriol. 194, 1619-1620. doi: 10.1128/JB.06749-11

Conflict of Interest Statement: The authors declare that the research was conducted in the absence of any commercial or financial relationships that could be construed as a potential conflict of interest.

Copyright (C) 2019 Ramirez, Penwell, Traglia, Zimbler, Gaddy, Nikolaidis, Arivett, Adams, Bonomo, Actis and Tolmasky. This is an open-access article distributed under the terms of the Creative Commons Attribution License (CC BY). The use, distribution or reproduction in other forums is permitted, provided the original author(s) and the copyright owner(s) are credited and that the original publication in this journal is cited, in accordance with accepted academic practice. No use, distribution or reproduction is permitted which does not comply with these terms. 